\title{
Modulating the TSH Receptor with High Specificity Gets a Step Closer Toward Use in Clinical Practice
}

\author{
Marius N. Stan ${ }^{1}$ and J. Paul Banga ${ }^{2}$ \\ ${ }^{1}$ Division of Endocrinology, Diabetes, and Metabolism; Department of Medicine, Mayo Clinic College of \\ Medicine and Science, Rochester, Minnesota, U.S.A. \\ 2Emeritus, King's College London, London, United Kingdom
}

Review of: Marcinkowski P, Hoyer I, Specker E, Furkert J, Rutz C, Neuenschwander M, Sobottka S, Sun H, Nazare M, Berchner-Pfannschmidt U, von Kries JP, Eckstein A, Schülein R, Krause G 2019 A new highly thyrotropin receptor-selective small-molecule antagonist with potential for the treatment of Graves' orbitopathy. Thyroid 29:111-123. PMID: 30351237.

\section{SUMMARY}

\section{Background}

Graves' disease (GD) is caused by autoantibodies (TRAbs) against the TSH receptor (TSHR). One of its most dreaded comorbidities is Graves' orbitopathy (GO), where TSHR expressed on orbital fibroblasts together with TRAbs play a pathogenic role. Current therapies for GD inhibit the synthesis of thyroid hormones (antithyroid drugs) or eliminate the thyroid (radioactive iodine treatment or surgery). GO therapy is focused on diminishing the inflammation (steroid therapy or selenium), followed by surgical rehabilitation. However, for both entities, targeting the TRAbs early action would be a more specific approach. The TSHR is the ideal target as the earliest step in modulating the disease process. Developing small-molecule antagonists (SMAs) for the TSHRs are thus highly desirable for treating GD and GO. The TSHR belongs to the G-proteincoupled receptor family (GPCRs), which include follicle-stimulating-hormone (FSH) and luteinizing hormone chorionic gonadotropin (LHCG) receptors. SMAs for the TSHRs have been described over time (1-3), but they also antagonized other GPCRs, making them unsuitable for clinical use. In contrast, the article we discuss here presents a new SMA with especially high efficacy and specificity for TSHRs (4).

\section{Methods}

A chemical library of bioactive molecules of 16,544 compounds was assessed individually by high-throughput screening to inhibit cellular TSH-induced CAMP production. Positive hits were further selected for secondary screening at varying concentrations, including screening to eliminate $\mathrm{TSH}$-independent inhibition. Final selection of one promising compound, termed S37, showed a molecule with seven chiral centers. Chemical synthesis of S37 and separation of its two diastereomers narrowed the active isomer to the single active compound S37a.

Other TSHR-signaling pathways were evaluated by inhibition of b-arrestin-1 recruitment after TSH stimulation, which is more prevalent in bone. Inhibition by S37 of TSHR stimulated by antibody ligands included the monoclonal antibodies with powerful agonist activity M22 (human) and KSAb1 (mouse). Serum samples from patients with GO positive for TRAbs were also included. Specificity of S37a was tested on human TSH-, FSH- or LHCG-receptortransfected cells stimulated with their appropriate ligand hormones. Other TSHR antagonists, ANTAG3 and K14, were similarly tested. Pharmacokinetic (PK) studies in mice were conducted by contract Company. 


\section{Results}

Identifying new TSHR SMA: High-throughput screening of chemical library compounds for their ability to inhibit $\mathrm{TSH}$-induced CAMP production identified 350 compounds. These were retested on a broad concentration range $(0.2-50 \mu \mathrm{M})$ and assessed for TSHR-independent inhibition of cAMP production. The 12 selected compounds were placed in HEK-TSHR-transfected cell cultures, where 2 compounds were able to inhibit TSH-induced cAMP accumulation by $>50 \%$. The lead compound selected was S4. Interestingly, only one of its diastereomers S37, with a noteworthy bent configuration, had an inhibitory effect (70\% at $50 \mu \mathrm{M})$. Testing its 2 enantiomers, $\mathrm{S} 37 \mathrm{a}$ and $\mathrm{S} 37 \mathrm{~b}$ revealed that S37a had a 20\% stronger inhibition than the parent compound S37. The other enantiomer S37b was a weak inhibitor. The TSHR can also signal by recruiting b-arrestin-1 recruitment, which was inhibited by S37a in a noncompetitive manner.

S37a specificity: S37a was tested in parallel with 2 other known TSHR antagonists (ANTAG3 and K14). S37a proved to have the highest inhibition for TSHR, with no significant impact on FSH and LHCG receptors, in stark contrast to ANTAG3 and K14.
S37a against patient's serum: In order to replicate the pathogenesis of hyperthyroidism and GO, S37a was tested against the potent TSHR-stimulating monoclonal antibodies M22 and KSAb1. S37a was able to inhibit the stimulatory action of these antibodies. This was again replicated when tested against the diverse TSHR-activating antibodies found in 10 patients with GO. The addition of S37a was able to inhibit up to $60 \%$ of the inhibitory effect.

Toxicity and pharmacokinetics: S37a added in cultures at concentrations twice of those tested for inhibitory action did not affect cell viability. It was also well tolerated in mice, without noticeable adverse effects. Moreover, in mice, S37a had an astonishing $53 \%$ bioavailability and a half-life of 3 hours at 10 $\mathrm{mg} / \mathrm{kg}$ of body weight.

\section{Conclusions}

These data prove that TSHR is extremely sensitive to the action of the new SMA S37a, which achieves excellent inhibition and specificity for this receptor. Its high degree of specificity is likely due to its bent shape. S37a could be ready for therapeutic testing in patients with GD and GO once specificity studies are completed and its potency optimized.

\section{COMMENTARY}

The developments reported here are exciting, yet at the same time, invite caution. TSHR is a therapeutic target for GD and GO that has yet to be in the crosshairs of our medical armamentarium. However, since TSHR belongs to GPCRs, the largest family of membrane receptors, a ligand to TSHR can potentially easily interact with similar but unintended targets in the same family of receptors. Such is the case with 2 other antagonists to TSHR, ANTAG3 and $\mathrm{K} 14$. Therefore, it is very important to see the evidence that S37a has good inhibition combined with excellent specificity against TSHR. However,
TSHR is present beyond the thyroid and orbit, in tissues such as bone, muscle, and adipose tissue, whose function in these extrathyroidal tissues remains to be fully determined. The result of antagonizing TSHR at these locations remains to be assessed in subsequent studies. Furthermore, it is important to note that S37a was tested against only a small number of serum samples from GO patients, where neither the subtype (stimulating, blocking, or neutral antibody to TSHR) nor the titer of anti-TSHR antibody was known. In addition, S37a was tested against a limited family of GPCRs, and its impact 
on other proteins and receptors will need to be evaluated in the future.

To maximize the benefit-risk ratio, additional investigation into the binding site of $37 a$ is required to allow further refinement of its inhibitory action as well as its specificity to TSHR. Importantly, S37a has potency for TSHR in the low micromolar rangefor clinical studies, it needs to be optimized as a nanomolar TSHR antagonist, as obtained for Org 274179-0 antagonist (1). It is also important to note that S37a binds downstream in the transmembrane domain (TMD) of TSHR. Thus, despite the variation in TSHR antibodies found in patients with GD and GO, S37a is likely to retain its inhibitory effect as these antibodies bind upstream from TMD and signal through the S37a-binding region.

Is there a need for these developments? Current therapies for GD and GO are less than perfect. GD therapy renders many patients permanently hypothyroid, with a lifelong need for thyroid hormone- replacement therapy and occasionally persistent impairment of quality of life (5). The use of antithyroid drugs can circumvent the hypothyroidism-related issues but raises the risk of some serious side effects early on and does nothing to prevent the risks of developing GO and pretibial dermopathy. SMAs like S37a can theoretically circumvent all these issues through their specific, scalable, and reversible blockade of action of TRAbs on TSHR. It could also become a desirable therapy in patients with GO, since it is intervening on an essential early step in the pathophysiology of the disease. Whether S37a action might be more efficient than the recently demonstrated action of a humanized monoclonal antibody targeting the IGF-1 receptor (6), or whether a combination of two would be able to quell even the most severe form of GO, remains to be seen. From a thyroidologist's perspective, this is an exciting time for testing new therapeutics, after several decades of "older" therapeutics of radioiodine or antithyroid drugs for treatment of this debilitating condition.

\section{REFERENCES}

1. Van Zeijl CJ, Van Koopen CJ, Surovtseva OV, De Gooyer ME, Plate R, Conti P, Karstens WJ, Timmers $M$, Saeed P, Wiersinga WM, et al. 2012 Complete inhibition of rhTSH-, Graves' disease IgG-, and M22-induced cAMP production in differentiated orbital fibroblasts by a low-molecular weight TSHR antagonist. J Clin Endocrinol Metab 97(5):E781-E785.

2. Neumann S, Nir EA, Eliseeva E, Huang W, Marugan J, Xiao J, Dulcey AE, Gershengorn MC 2014 A selective $\mathrm{TSH}$ receptor antagonist inhibits stimulation of thyroid function in female mice. Endocrinology 155:310-314.
3. Latif R, Realubit RB, Karan C, Mezei M, Davies TF 2016 TSH receptor signaling abrogation by a novel small molecule. Front Endocrinol (Lausanne) 7:130.

4. Marcinkowski P, Hoyer I, Specker E, Furkert J, Rutz C, Neuenschwander M, Sobottka S, Sun H, Nazare M, Berchner-Pfannschmidt U, et al. 2019 A new highly thyrotropin receptor-selective small-molecule antagonist with potential for the treatment of Graves' orbitopathy. Thyroid 29:111-123. 
Marius N. Stan and J. Paul Banga
5. Törring O, Watt T, Sjölin G, Byström K, AbrahamNordling M, Calissendorff J, Cramon PK, Filipsson Nyström H, Hallengren B, Holmberg M, et al. 2019 Impaired quality of life after radioiodine therapy compared to antithyroid drugs or surgical treatment for Graves' hyperthyroidism: a long-term follow-up with the thyroid-related patient-reported outcome questionnaire and 36-Item Short Form Health Status Survey. Thyroid 29:322-331.
6. Smith TJ, Kahaly GJ, Ezra DG, Fleming JC, Dailey RA, Tang RA, Harris GJ, Antonelli A, Salvi M, Goldberg RA, et al. 2017 Teprotumumab for thyroid-associated ophthalmopathy. N Engl J Med 376:1748-1761. 